\title{
Genetic analysis of osteopetrosis in Pakistani families identifies novel and known sequence variants
}

\author{
Chunyu Liu ${ }^{1 \dagger}$, Muhammad Ajmal2 ${ }^{2 \dagger}$, Zaineb Akram ${ }^{3}$, Tariq Ghafoor ${ }^{3}$, Muhammad Farhan ${ }^{3}$, Sobia Shafique ${ }^{2}$, \\ Sughra Wahid ${ }^{4}$, Shahar Bano ${ }^{4}$, Jianqiu Xiao ${ }^{5}$, Humayoon Shafique Satti ${ }^{6}$, Feng Zhang ${ }^{1 *}$ and \\ Tahir Naeem Khan ${ }^{6,7^{*}}$
}

\begin{abstract}
Osteopetrosis is a genetically heterogenous, fatal bone disorder characterized by increased bone density. Globally, various genetic causes are reported for osteopetrosis with all forms of inheritance patterns. A precise molecular diagnosis is necessary for prognosis and for prescribing treatment paradigms in osteopetrosis. Here we report on thirteen individuals diagnosed with infantile malignant osteopetrosis coming from ten unrelated Pakistani families; nine of whom are consanguineous. We performed whole exome sequencing and Sanger sequencing in all families and identified homozygous variants in genes previously reported for autosomal recessive inheritance of osteopetrosis. All the identified variants are expected to affect the stability or length of gene products except one nonsynonymous missense variant. TCIRG1 was found as a candidate causal gene in majority of the families. We report six novel variants; four in TCIRG1 and one each in CLCN7 and OSTM1. Our combined findings will be helpful in molecular diagnosis and genetic counselling of patients with osteopetrosis particularly in populations with high consanguinity.
\end{abstract}

Keywords: Autosomal recessive osteopetrosis, Genetic diagnosis, TCIRG1, Disease variants

\section{Introduction}

Osteopetrosis is a group of clinically and genetically heterogeneous disorders of increased bone density caused by defective osteoclast function and decreased bone resorption, making bones fragile and prone to fractures [1]. Several types of osteopetrosis have been described in literature, mainly distinguished on the basis of pattern of inheritance; autosomal dominant, autosomal recessive

\footnotetext{
*Correspondence: zhangfeng@fudan.edu.cn; tahir.khan@numspak.edu.pk ${ }^{\dagger}$ Chunyu Liu and Muhammad Ajmal have contributed equally to this work

1 Obstetrics and Gynecology Hospital, NHC Key Laboratory of Reproduction Regulation (Shanghai Institute for Biomedical and Pharmaceutical Technologies), School of Life Sciences, Fudan University, Shanghai 200011, China

${ }^{6}$ Department of Biological Sciences, National University of Medical Sciences, Rawalpindi 46000, Pakistan

Full list of author information is available at the end of the article
}

and X-linked or intermediate autosomal type [2]. Autosomal recessive osteopetrosis (ARO), also known as infantile malignant osteopetrosis is a rare phenotype with an estimated incidence of 1 in 250,000 live births [3] although as in the case with other autosomal recessive genetic disorders, the incidence of ARO is expected to be higher in highly consanguineous populations. The symptoms of ARO start appearing soon after birth and often proves to be fatal if not treated early. Symptoms arising from a reduced marrow compartment include anemia, thrombocytopenia and infections along with hepatosplenomegaly. An increased density of skull bone results in neurodegeneration and compression of optic nerve resulting in blindness and deafness [4].

ARO is caused by biallelic mutations in one of the at least seven genes namely TCIRG1, CLCN7, OSTM1, SNX10, PLEKHM1, TNFSF11, TNFRSF11A [5]. 
Mutations in genes TCIRG1, CLCN7, OSTM1, SNX10 and PLEKHM1 lead to osteoclast rich osteopetrosis, which has abundant but nonfunctional osteoclasts [6]. These genes encode proteins that are involved in acidification of the resorption lacunae and/or vascular transport. Mutations in TNFSF11 and TNFRSF11A result in blockage of osteoclastogenesis which is osteoclast poor ARO [7].

Approximately half of the ARO cases are caused by mutations in TCIRG1 followed by CLCN7 mutations which are associated with $17.5 \%$ of ARO cases [8]. More than hundred candidate disease causal variants have been reported in TCIRG1 (The Human Gene Mutation Database), majority in Western populations. The mutational spectrum of ARO is underreported in a highly consanguineous Pakistani population. In current study, we report genetic analysis of ARO in ten unrelated families originating from Pakistan.

\section{Methods}

\section{Human research participants}

This study includes total of 13 affected individuals from ten unrelated families afflicted with osteopetrosis. Nine of these families are consanguineous while for one family the consanguinity is not known. Study participants originate from different regions of Pakistan. Studies and procedures related to clinical examination, human sample collection and genetic analysis were approved by Institutional Review Boards and Ethical committees of the Armed Forces Bone Marrow Transplant Centre, Rawalpindi, Pakistan (families OP1, OP2, OP3, OP4) and Institute of Biomedical and Genetic Engineering, Islamabad, Pakistan (families OP5, OP6, OP7, OP8, OP9, OP10). Signed informed consent was obtained from all participants or their legal representatives for study procedures and publication of clinical and genetic findings. Subsequent to informed consent, we obtained peripheral blood samples from affected individuals and their healthy family members by standard venipuncture. We extracted DNA using a phenol-chloroform extraction method or QIAamp DNA Maxi Kit (Qiagen).

\section{Genetic analysis}

We performed whole exome sequencing (WES) in eight families, on genomic DNA obtained from an affected individual from each family except family OP4 where we performed WES in both affected individuals in the family. Genomic DNA was target enriched by the Agilent SureSelect ${ }^{\mathrm{XT}}$ Human All Exon Kit. Next-generation sequencing was conducted with the Illumina HiSeq X-TEN platform at Cloud Health Genomics. Reads were aligned to the human genome reference assembly (UCSC Genome Browser hg19) with BWA. Picard software was employed to remove PCR duplicates and evaluate the quality of variants to attaining effective reads, quality bases, average coverage depth and coverage ratio. Single-nucleotide variants (SNVs) and indels were called and analyzed with GATK using an in-house variant filtration pipeline. We then used ANNOVAR for functional annotation with OMIM, Gene Ontology, KEGG Pathway, SIFT, PolyPhen-2 and MutationTaster.

In WES-analyzed families, variants generated by WES were assessed and filtered for rare variants with a minor allele frequency (MAF) of $<0.01$ in relevant ethnicity-matched populations in control databases (including the Genome Aggregation Database; gno$\mathrm{mAD}$, the Exome Aggregation Consortium; ExAC, and the 1000 Genomes Project). Non-synonymous, exonic or splice variants, conforming to recessive pattern of inheritance of disease in each family were prioritized as candidates. We performed bidirectional Sanger sequencing with BigDye terminator v3.1 cycle sequencing chemistry to confirm candidate variants and segregation in all available family members.

In two additional families (OP5 and OP9), we performed bidirectional Sanger sequencing of previously published genes associated with autosomal recessive osteopetrosis starting from sequencing of the most frequently associated genes such as TCIRG1 and CLCN7. For this purpose, primers were designed covering all the exons and exon-intron boundaries of candidate genes. PCR amplified, purified samples were run on ABI 3500 Genetic Analyzer (Applied Biosystems) and sequence chromatogram was analyzed using Sequencing Analysis Software v6.0 (Applied Biosystems).

\section{Results \\ Clinical features of the osteopetrosis patients (Table 1)}

\section{OP1}

The affected child (OP1-2), deceased now, was born to consanguineous parents. He was the youngest of the three siblings. He was deaf and mute since birth and was diagnosed with osteopetrosis at the age of nine months, when he presented with progressive pallor, bicytopenia, hepatosplenomegaly, delayed developmental milestones and failure to thrive. Skeletal survey revealed generalized increase in bone density suggestive of marble bone disease. Due to severe anemia and thrombocytopenia, allogeneic stem cell transplantation was planned after HLA-matching with elder sister (OP1-1). However, the patient succumbed to infections and died before hematopoietic stem cell transplantation (HSCT). 


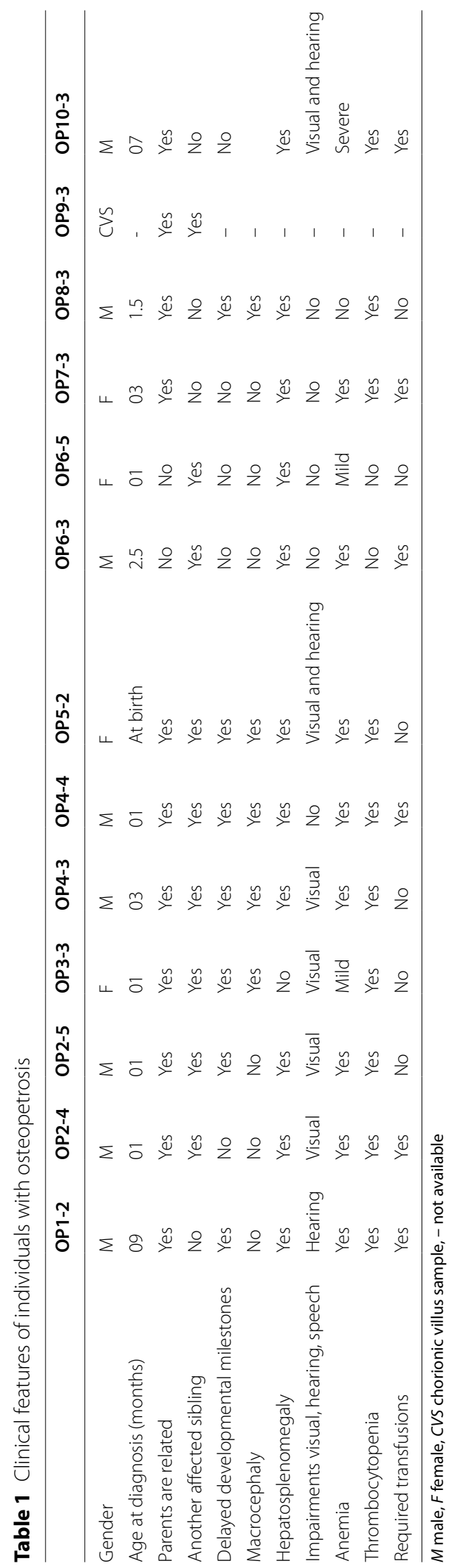




\section{OP2}

The parents in OP2 family are first-cousins. Their two male children (OP2-4 and OP2-5) are affected with infantile malignant osteopetrosis, whereas the eldest female sibling (OP2-3) is healthy. The elder of the two siblings (OP2-4) presented with severe anemia and bruises in the first month after birth. Bone marrow aspirate from OP2-4 revealed hypocellularity with depressed erythropoiesis. Skeletal survey showed increased bone density and was provisionally labelled as marble bone disease. Due to severe anemia and thrombocytopenia, he was receiving regular blood transfusion since then. He underwent splenectomy at the age of 5 years. He got HLA matched with the father. However, he died at the age of six and a half years before HSCT due to transfusion-associated sepsis.

Considering family history, when the youngest sibling (OP2-5) was born, his chest X-ray and X-ray of long bones were performed at age one month, which showed increased bone density and reduced medullary cavity, suggestive of osteopetrosis. His growth milestones are delayed for his age. He has poor vision, but has some perception of light, whereas hearing is intact. Though his blood parameters are on the lower side but he never received any transfusion. He got HLA-matched with the sister and underwent HSCT at the age of nine months. He is currently one year post-HSCT and off medication.

\section{OP3}

The parents in this family are first-cousins and they have two children affected with osteopetrosis. One of the affected child died at the age of three years due to complications of osteopetrosis. The details of clinical history are not available for the deceased child. In other affected child (OP3-3), skeletal scan confirmed marble bone disease. She has enlarged skull and delayed growth milestones. She suffers from poor dentition and have difficulty in chewing food. Her vision is markedly impaired, though she is able to follow light. Her blood counts are stable at slightly below normal limits and she has no blood transfusion history.

\section{OP4}

This consanguineously married couple has two children (OP4-3 and OP4-4) affected with osteopetrosis, currently aged four and two and a half years, respectively. Both children have delayed growth landmarks, frontal bossing of skull, proptosis, hepatosplenomegaly and bicytopenia. The elder sibling has bilateral blindness with intact hearing. Bone scan in both children revealed marble bone disease.

\section{OP5}

Family OP5 had two affected children, both of them deceased now. One male child died at an early age having severe symptoms of osteopetrosis. At the time of birth, he had macrocephaly, hepatosplenomegaly, blindness with exophthalmoses and deafness. He also presented with severe anemia due to bone marrow failure along with other respiratory problems, which possibly led to his demise. Genetic screening was not performed in this patient.

The second patient (OP5-2) in this family was a female baby who survived till the age of one year. She manifested feeding problems since her birth. Other clinical features of the osteopetrosis were also obvious from the time of birth. These included prominent macrocephaly, skeletal deformities including thick and dense skull bones, neurological abnormalities, frontal bossing and hypertelorism, nystagmus with complete visual loss, deafness, hepatosplenomegaly, bone marrow suppression resulting in severe cytopenia. The serum calcium level was markedly low. She eventually died due to hematological complications.

\section{OP6}

The family has two affected children born to non-consanguineous couple. Individual OP6-5, deceased now, initially presented with mild splenomegaly. Computed tomography scan of orbit and brain showed increased bone density in OP6-5 which was further confirmed by $\mathrm{X}$-ray scan showing generalized increase in bone density of all bones. Other clinical features include bone within bone appearance and vertebra within vertebra appearance in spine and dense skull bones with sclerotic base. His sample was collected for genetic screening, before he died at the age of 5 years.

Clinical assessment of second affected child (OP6-3) in this family reported occipitofrontal circumference of $43 \mathrm{~cm}$, proptosis, pallor, and no evidence of cranial nerve involvement. Massive hepatosplenomegaly was also observed; dentition was found to be normal for age. Her $\mathrm{X}$-ray scans confirmed increased bone density; indicative of osteopetrosis.

\section{OP7}

The parents in this family are first cousins. Their only child (OP7-3) deceased now, was diagnosed with osteopetrosis at the age of three months. She presented with pallor, bicytopenia and hepatosplenomegaly. She was initially screened for beta-thalassemia and was found heterozygous for $\mathrm{Cd} 15(\mathrm{G}>\mathrm{A})$ mutation in $H B B$ inherited from her healthy mother. Bone marrow biopsy revealed $2 \%$ blast cells and fragmented red blood cells. 
Radiological evidence was inconclusive for establishing diagnosis of osteopetrosis, therefore whole exome sequencing was performed in affected child to help diagnose the disease at molecular level.

\section{OP8}

The proband (OP8-3) in this family, deceased now, was born to consanguineous parents. Hematological investigations of OP8-3 showed lymphocytes with atypical morphology and nucleated red blood cells, decreased platelet count and increased reticulocyte count. This was accompanied by moderate hepatosplenomegaly. Radiological scan revealed increase bone density with metaphyseal lucent bands and increased skull size for age indicative of marble bone disease. No visual and hearing impairments were observed at the time of diagnosis.

\section{OP9}

The parents in this family are related to each other. Previously they had two children (both deceased) affected with osteopetrosis, but we could not gather detailed clinical history for them. The family came to us with a chorionic villus sample (CVS) from fetus for prenatal genetic screening of osteopetrosis. No clinical details were possible for OP9-3, as it was a fetal sample. We screened all three DNA samples (both parents and fetal sample) for variation in osteopetrosis candidate genes through bidirectional Sanger sequencing to identify the disease causing mutation in the family. Sanger sequencing identified a homozygous mutation (c.2416T > A) in CLCN7 in the DNA from CVS while both parents were found heterozygous for the said mutation. The fetus was later aborted due to findings consistent with osteopetrosis.

\section{OP10}

The proband (OP10-3) born to consanguineous parents is a seven month old baby boy, a diagnosed patient of osteopetrosis with congenital heart disease and atrial septal defect. X-ray scan of proband showed bone-inbone appearance with prominent dense bones and frontal bossing. Other findings included hepatosplenomegaly, progressive vision and hearing loss and bilateral nystagmus. Patient has history of anemia and blood transfusion.

\section{Genomic studies identify recessive variants in known osteopetrosis genes}

To identify the genetic cause(s) of osteopetrosis, we performed WES in one affected individual from families OP1, OP2, OP3, OP6, OP7, OP8, OP10 and two affected individuals in family OP4. We obtained a mean target coverage of $124.61-155.93$ with a mean of $93.2 \%$ of bases covered $>20 \mathrm{X}$ for all affected individuals (Additional file 1: Table 1).
We filtered rare variants that were homozygous and compound heterozygous in affected individuals using a $\mathrm{MAF}<1 \%$. None of the compound heterozygous changes fulfilled our filtration criteria. However, we identified homozygous changes in each family in at least one gene known to cause osteopetrosis in multiple studies. Additionally, disease candidate gene sequencing via Sanger sequencing in further two families (OP5 and OP9) with osteopetrosis identified two homozygous variants, one each in TCIRG1 and CLCN7.

In total, seven families were identified with six different homozygous variants in TCIRG1, two families with the same homozygous variant in CLCN7 and a family with homozygous variant in OSTM1 (Fig. 1). Parents of the affected cases were asymptomatic and where available for genetic analysis, were found to be heterozygous for identified variants. Based on the relevance of identified gene variants to phenotype, segregation with disease, population frequencies and absence of homozygous changes in the general population, we considered these variants as likely cause of osteopetrosis in our families. Variants identified in this study were further characterized based on the variant interpretation guidelines of The American College of Medical Genetics and Genomics [9] (Table 2).

\section{Discussion}

Here, we describe ten unrelated families with 13 affected individuals who exhibit clinical features of infantile malignant osteopetrosis and harbor likely pathogenic recessive variants in genes previously associated with osteopetrosis. In seven out of ten families (70\%), we identified TCIRG1 variants as the likely causal gene for ARO, consistent with the findings implicating TCIRG1 as the pathogenic gene in more than $50 \%$ of ARO cases [10, 11]. The loss of function variants identified in families OP2, OP4, OP8, OP10 and splice site variants in families OP5, OP6, OP7 are predicted to result in frameshift and premature stop codons. We were not able to study the functional consequences of these variants due to unavailability of patient derived mRNA and cells. We expect the resulting products to be targeted for degradation through nonsense mediated decay or, less likely, to lead to the generation of truncated proteins that lack essential functional domains. The protein disruptive consequences of these variants implicate a loss of function as the plausible disease causal mechanism. Characteristically, these rare disruptive variants received an increase likelihood of pathogenicity in our cases (Table 2). A missense variant (TCIRG1:p.Gly172Asp) of uncertain significance identified in family OP1 affects a highly conserved amino acid residue (Additional file 1: Fig. 1) and is located in functionally crucial V ATPase I domain of TCIRG1. This variant is absent in public databases (gnomAD, 1000 


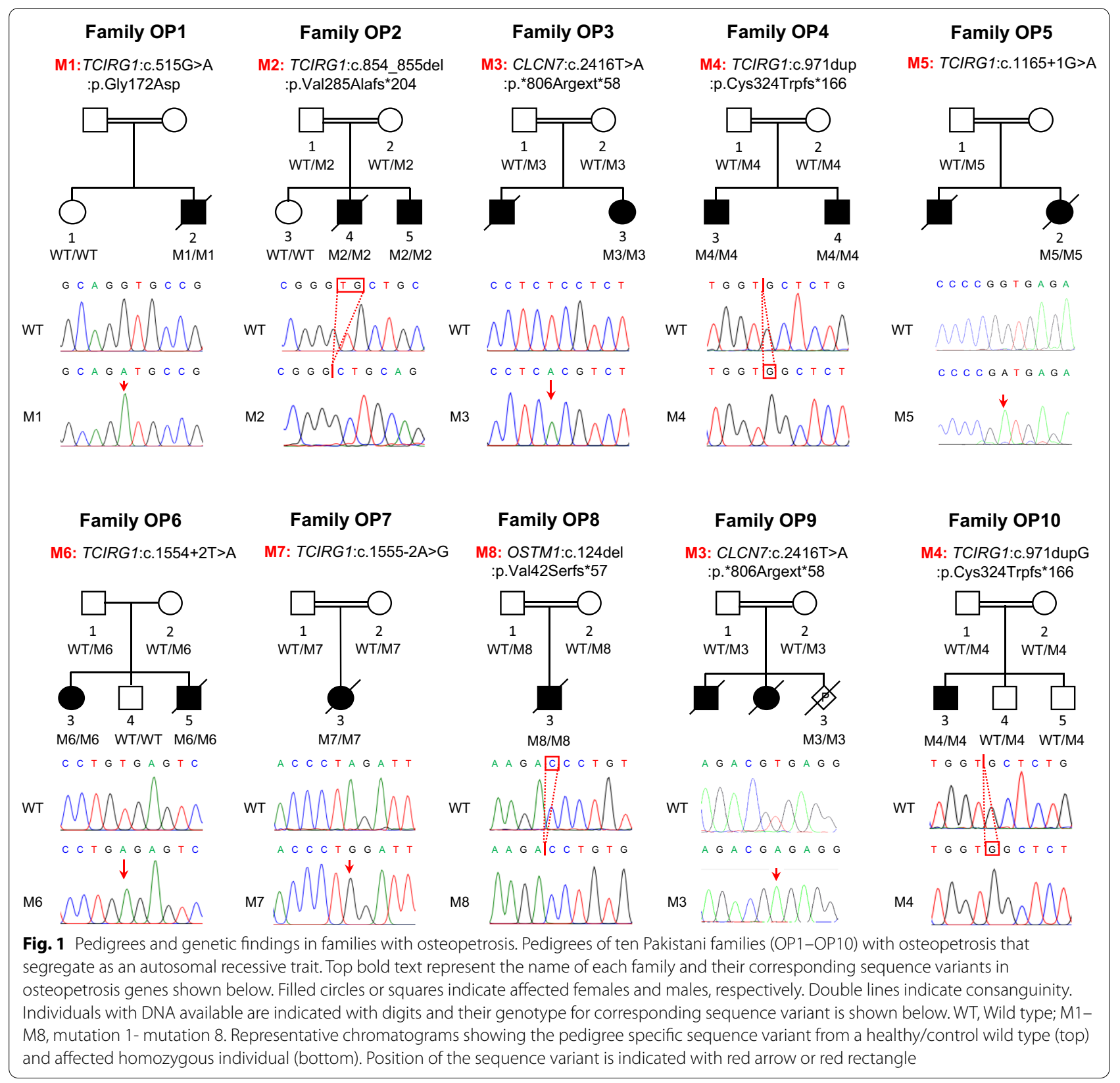

Genomes Project) and predicted to be functionally damaging (SIFT; damaging, PolyPhen-2; probably damaging). However, one should be cautious in interpreting this variant until confirmed in additional ARO cases. A nonsynonymous variant in CLCN7 (p."806Argext"58) identified in families OP3 and OP9 abolishes protein termination and expected to result in an elongated protein. We do not know the fate of this elongated protein however, CLCN7 functions as a membrane bound ion exchanger in a homodimer form [12, 13] and each abnormally elongated monomer may not adhere to conformational rearrangements required to form a membrane bound functional dimer for regulation of ion exchange. Additionally, CLCN7 forms a molecular complex with OSTM1 [14] and an elongated CLCN7 may lose the ability to form a functional complex. Further assays will be required to precisely delineate the functional consequences of CLCN7:p."806Argext"58 variant.

According to The Human Gene Mutation Database, more than 150 ARO causing mutations are reported in TCIRG1, distributed along the entire gene and comprising all mutation types such as, missense mutations, 


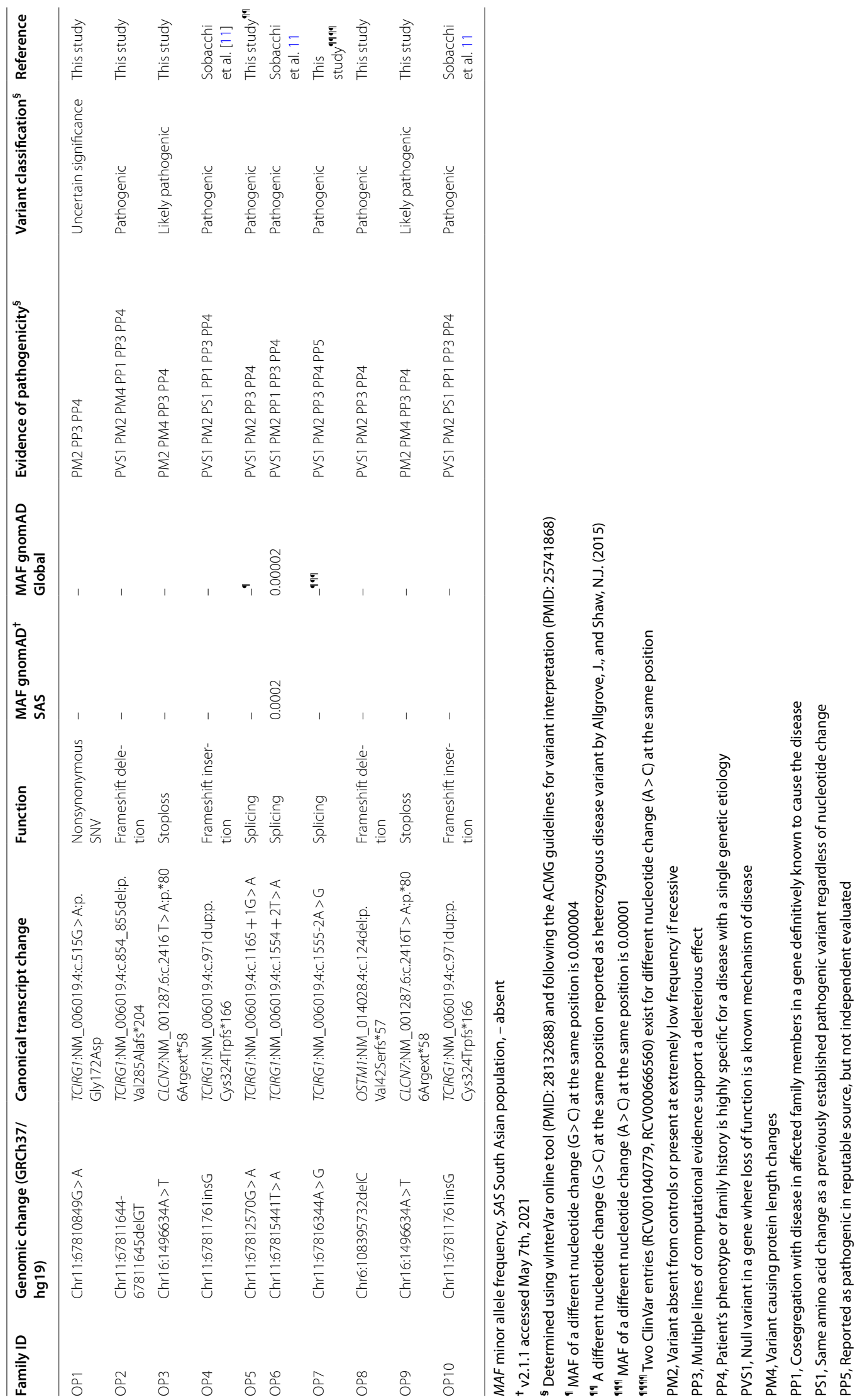


synonymous mutations, nonsense mutations, splice defects, deep intronic variants, small insertions and deletions and large deletions [8]. Given such a high mutational heterogeneity of TCIRG1 and absence of detailed phenotype description in some cohorts, it is difficult to establish a precise genotype-phenotype correlation in TCIRG1 deficient ARO. Notably, TCIRG1 mutations cause overall severe form of osteopetrosis indicated by severe hematological manifestations requiring HCST treatment. Mild to severe form of growth retardation and visual impairment are also frequently reported clinical features in TCIRG1 deficient ARO cohorts [2]. Consistent with the reported data, the patients carrying TCIRG1 mutations in our cohort manifest severe hematological complications and mild to severe visual impairment or normal vision at the time of diagnosis (Table 1). Similar clinical features of macrocephaly, hepatosplenomegaly and vision loss are reported in TCIRG1 deficient ARO cases from Pakistan [15].

Two families (OP3 and OP9) in our cohort are found to carry same stoploss variant in CLCN7 (c.2416 T>A:p."806Arg). Both families originate from two different geographic regions although belonging to same ethnic background (Punjabi). Both families are unknown to each other and are likely unrelated. The recurrence of stoploss variant in both families could be due to either a mutational hotspot or a founder effect. The latter possibility is supported by the observation that both families originate from same ethnic background. However, with only two affected families, we have limited data to establish the possibility of founder effect for this particular locus. Genetic investigations in additional ARO cases will be required to test this possibility. Previously, two homozygous missense mutations of CLCN7 segregating in cis in a Pakistani family with ARO was reported to exhibit dysmorphic facies, mild anemia, brain atrophy and bilateral optic atrophy [16]. Family OP3 has similar clinical features of delayed growth milestones, macrocephaly, visual impairment and mild anemia with no transfusion history. No clinical data is available for family OP9 since the tested DNA was obtained from CVS (Table 1).

Mutations in OSTM1 are relatively less frequently $(\sim 5 \%)$ reported in ARO cases [8]. OSTM1 mutations cause an osteoclast-rich, severe form of osteopetrosis with mild to severe hematological, ocular and growth defects [2]. In our cohort, only one family (OP8) carries a likely pathogenic variant in OSTM1 and presents with clinical features of severe hematological complications, mild hepatosplenomegaly and normal vision and hearing (Table 1).
In total, we report six novel sequence variants in autosomal recessive osteopetrosis; four in TCIRG1 and one each in CLCN7 and OSTM1. Our findings suggest that TCIRG1 is a major candidate for genetic screening of osteopetrosis in Pakistani population. Additionally, the variants identified in this study expand the mutational spectrum of autosomal recessive osteopetrosis, and will be of importance for molecular diagnosis and genetic counselling, particularly in populations with a high prevalence of autosomal recessive genetic disorders.

\section{Web resources}

1000 Genomes Project, https://www.internationalgenome. org.

Genome Aggregation Database (gnomAD), http://gnomad.broadinstitute.org.

The Human Gene Mutation Database (HGMD), http:// www.hgmd.cf.ac.uk.

\section{Supplementary Information}

The online version contains supplementary material available at https://doi. org/10.1186/s12920-021-01117-4.

Additional file 1. Quality of exome sequencing analysis and conservation analysis of TCIRG1:p.Gly172.

Acknowledgements

We are grateful to these ten families for their participation in our research.

Authors' contributions

All authors read and approved the final manuscript.

Funding

This work was supported by Grants from Higher Education Commission of Pakistan (NRPU), National Institute of Advance Studies and Research (NIASR/NUMS), National Natural Science Foundation of China (31625015 and 31521003), Shanghai Municipal Science and Technology Major Project (2017SHZDZX01), and the 111 Project (B13016). Above mentioned grants covered the costs of materials required for DNA extraction and expenses of whole exome sequencing and Sanger sequencing.

\section{Availability of data and materials}

The datasets used as a reference in whole exome sequencing are available in the human genome reference assembly (UCSC Genome Browser hg $19 \mathrm{https}: / /$ genome.ucsc.edu/). Reference sequences of gene transcripts described in current study NM_006019.4 (TCIRG1), NM_014028.4 (OSTM1) and NM_001287.6 (CLCN7) can be obtained from http://www.ensembl.org/. Details of the variants analyzed during the current study are deposited in Leiden Open Variation Database (https://databases.lovd.nl/) with screening IDs 377545 (TC IRG1:NM_006019.4:c.515G > A:p.Gly172Asp), 377546 (TCIRG1:NM_006019.4:C .854_855del:p.Val285Alafs*204), 377547 (CLCN7:NM_001287.6:c.2416T > A.p. *806Argext*58), 377548 (TCIRG1:NM_006019.4:c.971 dup:p.Cys324Trpfs*166), 377549 (TCIRG1:NM_006019.4:C.1165 + 1G > A), 377550 (TCIRG1:NM_006019 .4:C.1554+2T>A), 377551 (TCIRG1:NM_006019.4:C.1555-2A>G) and 377529 (OSTM1:NM 014028.4:c.124del:p.Val42Serfs*57). The raw datasets of family members generated during the current study are not publicly available due to constraints based on informed consent, ethical concerns and institutional policy. Access to the raw sequencing data can be requested through the corresponding authors; Feng Zhang or Tahir Naeem Khan. 


\section{Declarations}

\section{Ethics approval and consent to participate}

Legal guardians of the patients (parents) gave consent for participation in this study and for publication of data without disclosing their identity. Ethical aspects of the studies and procedures related to clinical examination, human sample collection and genetic analysis were approved by Institutional Reviews Boards and Ethical committees of participating institutes (see "Methods" section): Armed Forces Bone Marrow Transplant Centre, Rawalpindi, Pakistan (families OP1, OP2, OP3, OP4) and Institute of Biomedical and Genetic Engineering, Islamabad, Pakistan. All methods were performed in accordance with the relevant guidelines and regulations of The Declaration of Helsinki.

\section{Consent for publication}

Written consent to publish study findings was obtained from legal guardians of the affected individuals. Probands in current study are children under 17 years of age therefore consent to publish was collected from the parents/ guardians. The purpose of the study and its importance to patients and scientific community was explained to the parents of the affected individuals in their local language by the clinicians. The guardians gave written consent without revealing their identity in publication. Clinicians involved in the disease diagnosis coordinated the consent to Pl and research team for genetic investigations

\section{Competing interests}

The authors declare no competing interests.

\section{Author details}

'Obstetrics and Gynecology Hospital, NHC Key Laboratory of Reproduction Regulation (Shanghai Institute for Biomedical and Pharmaceutical Technologies), School of Life Sciences, Fudan University, Shanghai 200011, China. ${ }^{2}$ Institute of Biomedical and Genetic Engineering, Islamabad 44000, Pakistan. ${ }^{3}$ Armed Forces Bone Marrow Transplant Centre, CMH Medical Complex, Rawalpindi 46000, Pakistan. ${ }^{4}$ KRL General Hospital, Islamabad 44000, Pakistan. ${ }^{5}$ Division of Bone and Mineral Diseases, Musculoskeletal Research Center, Washington University School of Medicine, St. Louis, MO 63110, USA. ${ }^{6}$ Department of Biological Sciences, National University of Medical Sciences, Rawalpindi 46000, Pakistan. ${ }^{7}$ National Institute of Advance Studies and Research, National University of Medical Sciences, Rawalpindi 46000, Pakistan.

Received: 8 June 2021 Accepted: 26 October 2021

Published online: 09 November 2021

\section{References}

1. Wu CC, Econs MJ, DiMeglio LA, Insogna KL, Levine MA, Orchard PJ, Miller WP, Petryk A, Rush ET, Shoback DM, et al. Diagnosis and management of osteopetrosis: consensus guidelines from the osteopetrosis working group. J Clin Endocrinol Metab. 2017:102:3111-23.

2. Penna S, Capo V, Palagano E, Sobacchi C, Villa A. One disease, many genes: implications for the treatment of osteopetroses. Front Endocrinol. 2019;10:85

3. Sobacchi C, Schulz A, Coxon FP, Villa A, Helfrich MH. Osteopetrosis: genetics, treatment and new insights into osteoclast function. Nat Rev Endocrinol. 2013;9:522-36.
4. Chavez-Guitron LE, Ceron-Torres T, Sobacchi C, Ochoa-Ruiz E, VillegasHuesca S. Autosomal recessive osteopetrosis type I: description of pathogenic variant of TCIRG1 gene. Boletin Med Hosp Infantil Mex. 2018;75:255-9.

5. Oosterhof N, Chang IJ, Karimiani EG, Kuil LE, Jensen DM, Daza R, Young E, Astle L, van der Linde HC, Shivaram GM, et al. Homozygous mutations in CSF1R cause a pediatric-onset leukoencephalopathy and can result in congenital absence of microglia. Am J Hum Genet. 2019;104:936-47.

6. Sobacchi C, Villa A, Schulz A, Kornak U. CLCN7-Related Osteopetrosis. In: Adam MP, Ardinger HH, Pagon RA, Wallace SE, Bean LJH, Mirzaa G, Amemiya A, editors. GeneReviews((R)). Seattle; 1993.

7. Villa A, Guerrini MM, Cassani B, Pangrazio A, Sobacchi C. Infantile malignant, autosomal recessive osteopetrosis: the rich and the poor. Calcif Tissue Int. 2009:84:1-12.

8. Palagano E, Menale C, Sobacchi C, Villa A. Genetics of osteopetrosis. Curr Osteoporos Rep. 2018;16:13-25.

9. Richards S, Aziz N, Bale S, Bick D, Das S, Gastier-Foster J, Grody WW, Hegde M, Lyon E, Spector E, et al. Standards and guidelines for the interpretation of sequence variants: a joint consensus recommendation of the American College of Medical Genetics and Genomics and the Association for Molecular Pathology. Genet Med Off J Am Coll Med Genet. 2015;17:405-24.

10. Pangrazio A, Caldana ME, Lo lacono N, Mantero S, Vezzoni P, Villa A, Sobacchi C. Autosomal recessive osteopetrosis: report of 41 novel mutations in the TCIRG1 gene and diagnostic implications. Osteopor Int J Establ Res Cooper Eur Found Osteopor Natl Osteopor Found USA. 2012:23:2713-8.

11. Sobacchi C, Frattini A, Orchard P, Porras O, Tezcan I, Andolina M, BabulHirji R, Baric I, Canham N, Chitayat D, et al. The mutational spectrum of human malignant autosomal recessive osteopetrosis. Hum Mol Genet. 2001;10:1767-73.

12. Middleton RE, Pheasant DJ, Miller C. Homodimeric architecture of a CICtype chloride ion channel. Nature. 1996;383:337-40.

13. Leisle L, Ludwig CF, Wagner FA, Jentsch TJ, Stauber T. CIC-7 is a slowly voltage-gated $2 \mathrm{Cl}(-) / 1 \mathrm{H}(+)$-exchanger and requires Ostm 1 for transport activity. EMBO J. 2011;30:2140-52.

14. Lange PF, Wartosch $L$, Jentsch TJ, Fuhrmann JC. CIC-7 requires Ostm 1 as a beta-subunit to support bone resorption and lysosomal function. Nature. 2006;440:220-3.

15. Ajmal M, Mir A, Wahid S, Khor CC, Foo JN, Siddiqi S, Kauser M, Malik SA, Nasir M. Identification and in silico characterization of a novel p.P208PfsX1 mutation in V-ATPase a3 subunit associated with autosomal recessive osteopetrosis in a Pakistani family. BMC Med Genet. 2017:18:148.

16. Khan MA, Ullah A, Naeem M. Whole exome sequencing identified two novel homozygous missense variants in the same codon of CLCN7 underlying autosomal recessive infantile malignant osteopetrosis in a Pakistani family. Mol Biol Rep. 2018;45:565-70.

\section{Publisher's Note}

Springer Nature remains neutral with regard to jurisdictional claims in published maps and institutional affiliations.

Ready to submit your research? Choose BMC and benefit from

- fast, convenient online submission

- thorough peer review by experienced researchers in your field

- rapid publication on acceptance

- support for research data, including large and complex data types

- gold Open Access which fosters wider collaboration and increased citations

- maximum visibility for your research: over 100M website views per year

At BMC, research is always in progress.

Learn more biomedcentral.com/submissions 